\title{
AN INVESTIGATION OF CORNER SEPARATION \\ WITHIN A THRUST AUGMENTER HAVING COANDA JETS*
}

M. R. Seiler

Rockwe11 International

Columbus Aircraft Division

- Abstract -

An investigation was conducted to determine the way separation develops in the corners of thrust augmenter wings having Coanda jets. Hot film surface sensors and pressure transducers were used, and the results indicated that separation on the test augmenter began at a corner very close to the augmenter exit and then rapidly proceeded upstream. Measurements of the pressure fields in the corner region indicated that a modified form of the Stratford criterion could be used to predict the onset of scparation. Testing was conducted over a range of nozzle pressure ratios, aspect ratios, diffuser angles and designs of the boundary layer and roanda nozzles.

\footnotetext{
*Research supported by the Naval Air Development Center, Contract N6 2269-76-C-0402, Final Report No. NADC 76153-30
} 


\section{Introduction}

Thrust augmenters have been used in aircraft applications for a number of years. One of the first applications was to use them to draw cooling air over a jet engine nozzle. 1 Modest increases in thrust were also observed. During the early 1960's thrust augmenters were used to provide lift for the $\mathrm{XV}-4 \mathrm{~A}$ research VTOL aircraft. More recently 2 they have been used in the design concept of thrust augmenter wings (TAW) for direct lift in the Navy XFV-12A. Experimentally it has been observed that flow separation within the augmenter diffuser is often the limiting factor. The purposs of this study was to conduct a suitable testing program and analysis of an unswept, untapered (rectangular), model augmenter so that a preliminary separation criterion could be established.

\section{Approach}

The type of augmenter under consideration is one having a centerjet and two Coanda jets, as shown in Figure 1. The Coanda jets originate up:tream of the throat and provide a wall jet through the diffuser section. Without these Coanda jets, diffuser half-angles are limited to $\delta_{D} \approx .1$ radian to prevent separation. Small, boundary layer control (BLC) blowers were also mounted through the endwall and could be rotated manually to direct flow parallel to the diffuser flap. The end elements of the crossslot centerbody also direct some flow onto the endwalls to accomplish a BIC: function. Figure 2 shows a photograph of the test augmenter looking into the exit.

Flow to cach of the major augmenter components was measured by a scparate venturi and nozzle pressures were recorded by a total pressure probe at the nozzle exit. Nozzle pressure ratios were varied between 1.5 and 2.5 . The entire $50 \mathrm{~cm}\left(20^{\prime \prime}\right)$ span augmenter was mounted on a horizontal cradle suspended by four tie-rods attached to a rigid frame. Two 500-pound load cells measured the thrust. The tares of the flexible hoses, which deliver the primary air, were recorded versus supply pressure and removed from the measured thrust. A $4500 \mathrm{hp}$ Ingersoll-Rand compressor was used as the continuous air supply.

Experience has shown that flow separation generally occurs at or near a corner formed by the diffuser and the endwall. "There are two possible modes through which separation might develop (Figure 3 ). In the first mode separation would initiate at the augmenter exit and, because of the adverse pressure gradient within the diffuser, rapidly progress upstream. A cecond possible mode would be for separation to begin in the corner on the highly stressed Coanda surface and then proceed downstream until the ont irc diffuser corner was involved.

The type of BLC being used may have an effect on the separation mode and the angle at which separation occurs. The aspect ratio (AR = span/throat 
width) also effects the separation angle. 4 Finally, a reasonable data base should include testing over a wide nozzle pressure range.

The objective of the study was then to accomplish the following tasks:

a) To determine which of the two possible corner separation modes actually occur in an operating augmenter with Coanda jets.

b) l'o measure the pressure and velocity fields in the vicinity of the separation point for a range of nozzle pressure ratios $\left(P_{R}\right)$ and $B L C$ conditions at and near separated flow conditions.

c) To alter the augmenter $\mathrm{AR}$ and repeat task $\mathrm{b}$, above.

d) T'o alter the Coanda design to provide comparative data on Coandas of smaller $\mathrm{R} / \mathrm{t}$. This provides a more highly stressed coanda surface. In addition, the internal Coanda nozzle configurations were altered to examine the possible effects of exit velocity profile on separation.

c) To analyze the data to derive a separation criteria.

\section{Results}

For all tests the throat width, flap length, centerbody and BLC were as shown in Figure 1. Three different Coanda configurations, shown in lisure 4 , were used. The first, called a reference profile, maintained a Coanda radius to nozzle gap ratio of 26.5 . The ratio of augmenter throat area lo total nozzle area was $A_{2} / A_{0}=20.5$. This reference Coanda was $u$ sed in the study of separation mode and to provide a baseline augmentation ratio versus diffusion ratio, $\mathrm{A}_{3} / \mathrm{A}_{2}$.

Scparation Mode

The augmenter was instrumented as shown in Figure 5. Two flushmounted Thermo-Systems, Inc., Model 1237 hot film sensors were mounted on one flap surface at the endwall corner $.032 \mathrm{~cm}$ and $5.01 \mathrm{~cm}$ upstream from the flap trailing edge. Two Statham \pm 2 psi differential pressure transducers were connected to surface pressure taps similarly located on the opposite flap. The hot film sensors were connected to a model $1.050-2 \mathrm{C}$ Thermo-Systems, Inc., dual channel constant temperature anemometer whose output, together with that of the two transducers, was connected to a multi-channel Consolidated Electronics Corporation oscil$\log \mathrm{raph}$.

The response time of the hot-film sensor $\approx 5 \times 10^{-6} \mathrm{sec}$ ) was an order of magnitude faster than any mean flow changes likely to occur within the augmenter. Jsing the A.C. anemometer output, the diffuser angle, "D, was gradually increased to a point where a slight buffeting could be 
detected audibly (incipient separation). As shown in Figure 6 the turbulence level increased suddenly at the downstream sensor. Next the augmenter $\delta_{n}$ was rapidly raised beyond the point of attached flow and the two signals were displayed on the oscil liscope. Turbulence levels on the upstream sensor increased markedly within .0023 to .0027 seconds after the downstream sensor showed a similar increase. These tests, done at a nozzle pressure ratio of 2.0 , show that the separation was initiating downstream. Similar results were obtained at a pressure ratio of 1.5 .

\section{Augmentation Ratio}

The augmentation ratio is defined:

$$
\emptyset=\frac{\text { measured load-stand thrust }}{\text { ideal thrust from all primary jets and BLC }}
$$

where the ideal thrust uses the measured venturi mass flow and the isentropic nozzle velocity (expanded to atmospheric pressure). Using the reference profile Coandas, the results of Figure 7 were obtained with aspect ratio $=4.1$. Notice that separation occurs at a half angle of .21 radian without BLC and at .35 radian with BLC. When the aspect ratio is changed to 2.5 , Figure 8 shows that the overall $\emptyset$ levels are similar but separation occurs at a slightly lower diffuser angle. $A_{2} / A_{0}$ was 20.5 . BIC blowers were manually adjusted to blow parallel to the flaps.

Subsequent tests at $A_{2} / A_{0}=17$ were made on the top-hat profile and the vortex profile Coandas of Figure 4. The top-hat was designed to achieve a uniform velocity profile at the nozzle but $R / t$ was reduced to 9.3. The vortex profile Coanda was intended to produce a nozzle velocity that was greatest on the inner radius.

Figure 9 shows results of augmentation ratio versus $A_{3} / A_{2}$ for the top-hat profile. Although the initial slope, $\emptyset \mathrm{vs} \mathrm{A}_{3} / \mathrm{A}_{2}$, is similar to the reference profile, flow separation in the diffuser corner limits the performance to lower values of $\varnothing$.

Similar behavior was also noted on the vortex profile Coanda (Figure 10). At nozzle pressure ratios of 2.5 , corners became more difficult to attach on both of these Coanda shapes.

\section{Pressure Measurements}

A serios of 13 flush-mounted static taps were added in one corner of the diffuser near the exit, as shown in Figure 11. These taps were comnected to a water manometer and recorded during operation at all diffuser angles. Also recorded was the throat secondary static pressurc. lifirur 12 shows the location of the probe. 
Pigures 13 and 14 show the static pressure profiles in the diffuser corner for the reference profile Coanda. These measurements were taken at the diffuser angle for incipient separation, which also corresponds to the angle for maximum $\emptyset$. Figure 13 gives results without $B L C$ for an aspect ratio of 4.1. Figure 1.4 is for full BLC; that is, the BLC nozzle pressure was set equal to the Coanda and centerjet pressures. Also shown in the figures are the calculated term $\mathrm{xdp} / \mathrm{dx}$ exit, which is derived from the gradient of the pressure readings. The trend is toward a steeper gradient at the diffuser exit as the BLC is applied. Nominal $\mathrm{A}_{2} / \mathrm{A}_{0}$ was 20.5 .

Figures 15 and 16 show results for the top-hat and vortex profile Coandas at an aspect ratio of 4.1. $A_{2} / A_{0}$ was 17 . Notice that the static pressures are more negative than for the reference profile and the gradient is more steep. This is related to the increased Coanda nozzle gap and the reduced $A_{2} / A_{0}$.

\section{Separation Criteria}

One of the more successful airfoil separation criteria and the one considered herein is that of Stratford ${ }^{5}$ where the criteria is expressed as a non-dimensional number NST,

$$
\mathrm{N}_{S T}=\frac{C_{P}\left(x_{d} C_{P} / d x\right)^{1 / 2}}{\left(R_{N} \times 10^{-6}\right)^{1 / 10}}
$$

where $C_{p}$ is the pressure coefficient, defined by

$$
C_{P}=\frac{P(x)-P(0)}{q(0)}
$$

(.) i. based upon the difference between local wall static pressure $\mathrm{P}(\mathrm{x})$ and that pressure occurring at the start of the interaction region, $\mathrm{P}(0)$, at $x=0 . q(0)$ is the dynamic pressure $1 / 2 \rho U_{\max }^{2}$, where $U_{\max }$ is the maximum velocity at $x=0$. $R_{N}$ is the Reynolds number based upon $U_{\max }$ and $x$. Stratford's method involves an approximate solution of the equations of motion, and matching the solutions at the junction of the "inner" and "outer" boundary layer. A subsonic airfoil will not separate if $\mathrm{NST}_{\mathrm{ST}}<.37$.

Although Stratford used $P(0)$ as the wall pressure at $x=0$, there are experimental difficulties in determining its value accurately on a Coanda radius at choked pressures. Furthermore, because of the highly curved flow noar the Coanda, the value of $\mathrm{P}(0)$ at the wall is also difricult to predict analytically. For these reasons, $P(0)$ was chosen for the ejector diffuser to be the value of the static pressure in the uniform sccondary stream (see Figures 12 and 17 ). 
${ }_{9}(0)$ is merely a normalizing factor for the other pressure terms. Rather than take $q(0)=1 / 2 \rho \mathrm{U}_{\max }^{2}$ at the throat, it seemed correct in the high Mach number flow to use $q(0)$ as the maximum gage total pressure. Experience has shown that all static pressures (gage) in an augmenter can be normalized by nozzle gage total pressure. In a corner near the throat, the maximum value of $q$ is either:

(1) The gage total pressure set on the BLC blower, or

(2) The gage total pressure of the Coanda flow.

The greater of the above two quantities was used to set q(0). With no BlC turned on, the Coanda flow sets $q(0)$. With full BLC, the BLC nozzle pressure determines $q(0)$. Since $R_{N}$ must use the maximum velocity Unax, the isentropic flow equations were used to determine the relationship between $U_{\max }$ and $q(0)$ (see Figure 18).

$$
\begin{aligned}
\mathrm{U}_{\max } & =\left(2 \mathrm{RT} \frac{\gamma}{\gamma-1}\left(1-\mathrm{P}_{\mathrm{R}}-\frac{\gamma-1}{\gamma}\right)\right)^{1 / 2} \text { meters } / \mathrm{sec} \\
\mathrm{K} & =287 \text { Joules } /{ }^{\circ} \mathrm{K}-\mathrm{kg} \\
\gamma & =1.4 \\
\mathrm{~T} & \left.=\text { temperature, }{ }^{\circ} \mathrm{K} \text { (nominally } 290^{\circ} \mathrm{K}\right) \\
\mathrm{P}_{\mathrm{R}} & =\left(\mathrm{q}(0)+\mathrm{P}_{\infty}\right) / \mathrm{P}_{\infty} \\
\mathrm{P}_{\infty} & =\text { barometric pressure (nominally } 99 \text { Kilopascals) }
\end{aligned}
$$

I'able: I presents a summary of the Stratford number calculations for the augmenters constructed under the present study. There are three BLC conditions--full, minimum and no BLC for aspect ratios of 4.1 and 2.5 using the reference Coandas. Also included are the top-hat and vortex profile Coanda results. The table gives the nominal pressure ratio and the llap angle where the measurements and calculations were made.

Il is instructive to consider the difference in the three augmenters and $t o$ try to visualize what mechanism is setting NST . .02 as a conmon upper limit. Figure 19 shows a plot of the term $\mathrm{D}_{\infty}-\mathrm{P}(0) \cdots \mathrm{P}(\mathrm{x})-\mathrm{P}(0)$ and the term $x d P / d x$ versus diffuser angle for the three augmenters. The conditions are full BLC and $P R=2.0$. Notice that the vortex and tophat profiles produce larger values of $\mathrm{xdP} / \mathrm{dx}$ than does the reference profile. This, as mentioned earlier, is related to the larger nozzle gap and decreased $A_{2} / A_{0}$. The throat static gage pressurc, or its negative, $P_{o s}-P(0)$, is also greater for the vortex and top-hat at small diffuser angles. This is due to the reduced overall $A_{2} / A_{O}$. Finally near .175 to .2 radian, the reference profile produces the largest values of $x \mathrm{dP} / \mathrm{dx}$ and $\mathrm{P}_{\infty}-\mathrm{P}(0)$. The reference profile also produces the greatest

A lesson to be learned from Figure 19 is that a high $\emptyset$ augmenter should produce a large drop in throat static pressure (as is well known) but simultaneously must not produce a large value of $\mathrm{xdP} / \mathrm{dx}$ at the exit. 
This implies that small primary nozzles should be used to achieve wellmixed flows and nearly ambient static pressures at the exit. In other words, the exit static pressure should be nearly recovered to ambient. These facts are entirely consistent with the experience of many worker: in the area of thrust augmentation.

Figure 20 shows the calculated values of NST for these augmenters under the same operating conditions; i.e., full BLC and PR $=2.0$. The Stratford number rises to a maximum as flap angle is increased and does provide a useful separation criteria.

These plots indicate that we have not mistakenly selected a criteria this is insensitive to flap angle. The flow will be stable and attached provided

$$
\mathrm{N}_{\mathrm{ST}}=.0196
$$

Fior flap angles that produce separation, the Stratford number has no meining; that is, the criteria is to be used only in the range of $f l a p$ angles where $\mathrm{d} \mathrm{N}_{\mathrm{ST}} / \mathrm{d} \delta$ is positive.

It should be noted that some care in selecting BLC orientation is necded if these experiments are to be repeated. As stated earlier the BLC tubes were rotated manually to direct flow parallel to the flap. If this is not done, the unusual exit pressure profiles of Figure 21 will be obtained. Case 1 is caused by directing the BLC flow into the flap. It likely represents a helical flow pattern in the corner. Case 2 is similar with the opposite flap attached. Case 3 is the profile most like those of this study, with BLC blowing parallel to the flap. Case 4 is a separated flap.

\section{Conclusions}

1. Corner separation of the test thrust augmenting wing-type augmonter initiates at or near the augmenter diffuser exit and then rapidly progresses upstream until the whole corner from the vicinity of the augmenter throat to the exit is involved.

2. A modified form of the Stratford airfoil stall criterion successfully correlates the onset of augmenter separation in the test augmenter where the independent test variables were nozzle pressure ratio, augnenter aspect ratio, boundary layer control blower pressure ratio and Coanda configuration. The modification consists of a change in reference pressurc, $\mathrm{P}(0)$, and in definition of $\mathrm{q}$.

3. Circular Coandas with small $\mathrm{R} / \mathrm{t}$ cause separation to occur at lower diffuser angles. 
1. Greathouse, W. K., "Preliminary Investigation of Pumping and Thrust Characteristics of Full-size Cooling Air Ejectors at Several Exhaust-Gas Temperatures," NACA RM E54A18, 1954.

2. Throndson, L. W., "Compound Ejector Thrust Augmentation Development," ASME. 73-GT-67, 1973.

3. "Three-Dimensional Effects on Augmenters," Rockwell International Report NR76H-36, 1976.

4. Stewart, V. R., "A Study of Scale Effects on Thrust Augmenting Ejectors," Rockwell International Report NR76H-2, 1976.

5. Stratford, D. S., "The Predictions of Separation of the Turbulent Boundary Layer," J. Fluid Mechanics, p. 1, January 1959. 


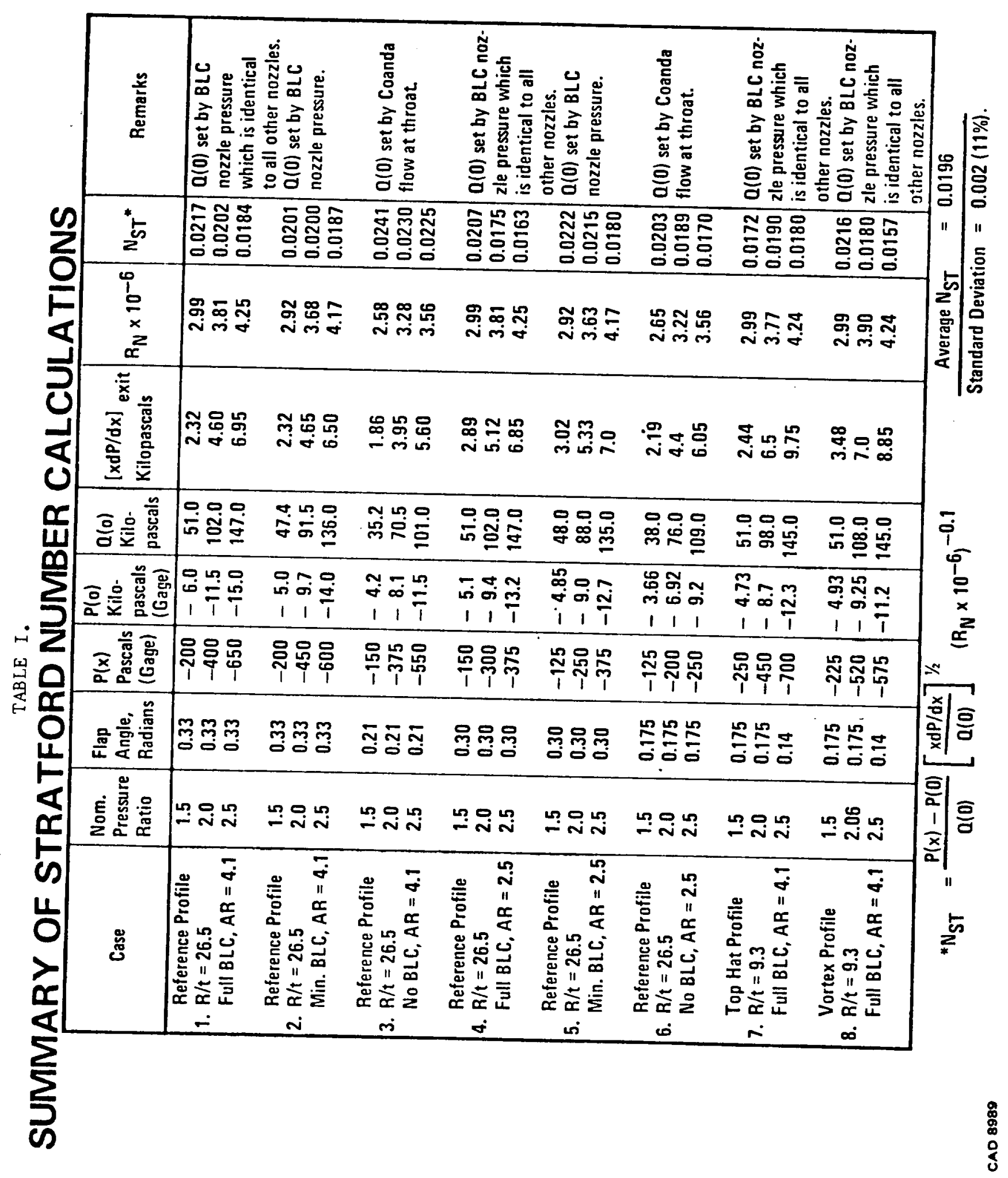




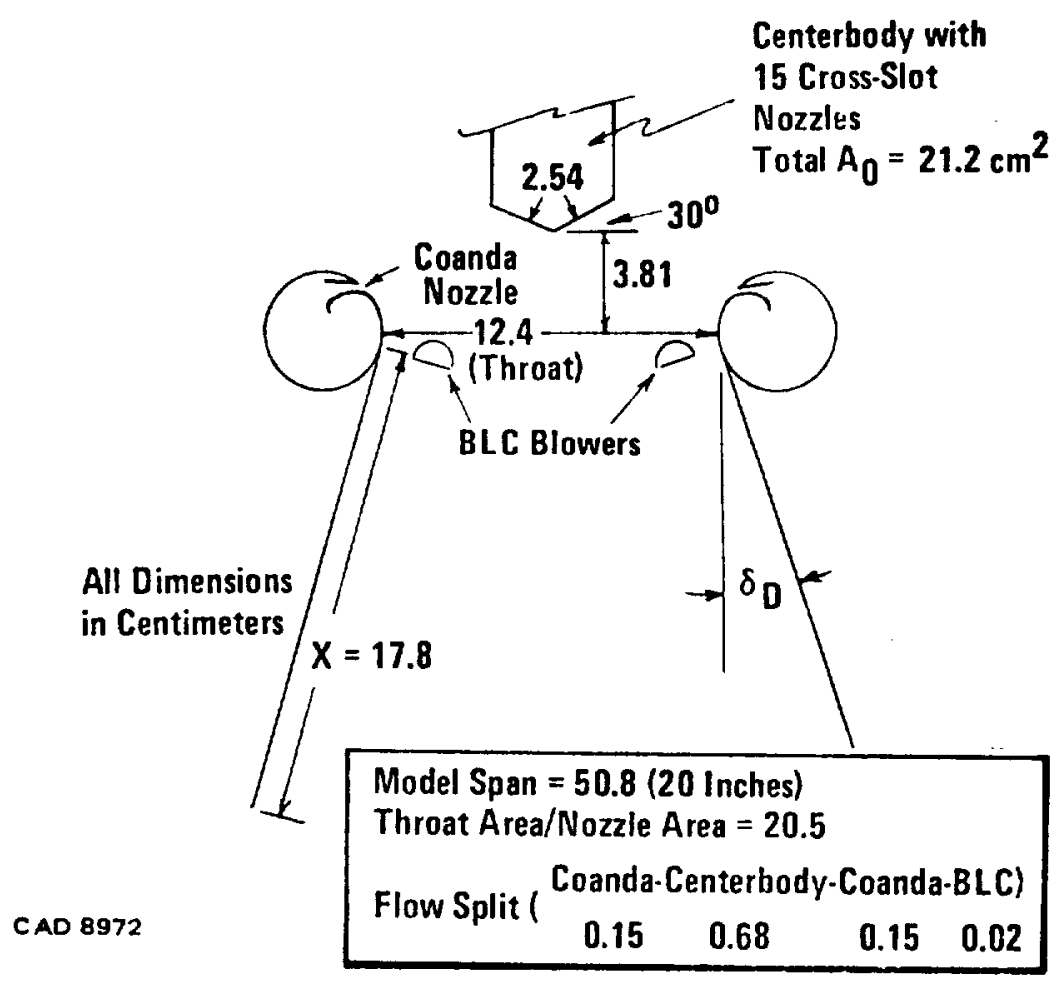

Figure 1. Sectional view of test augmenter. 


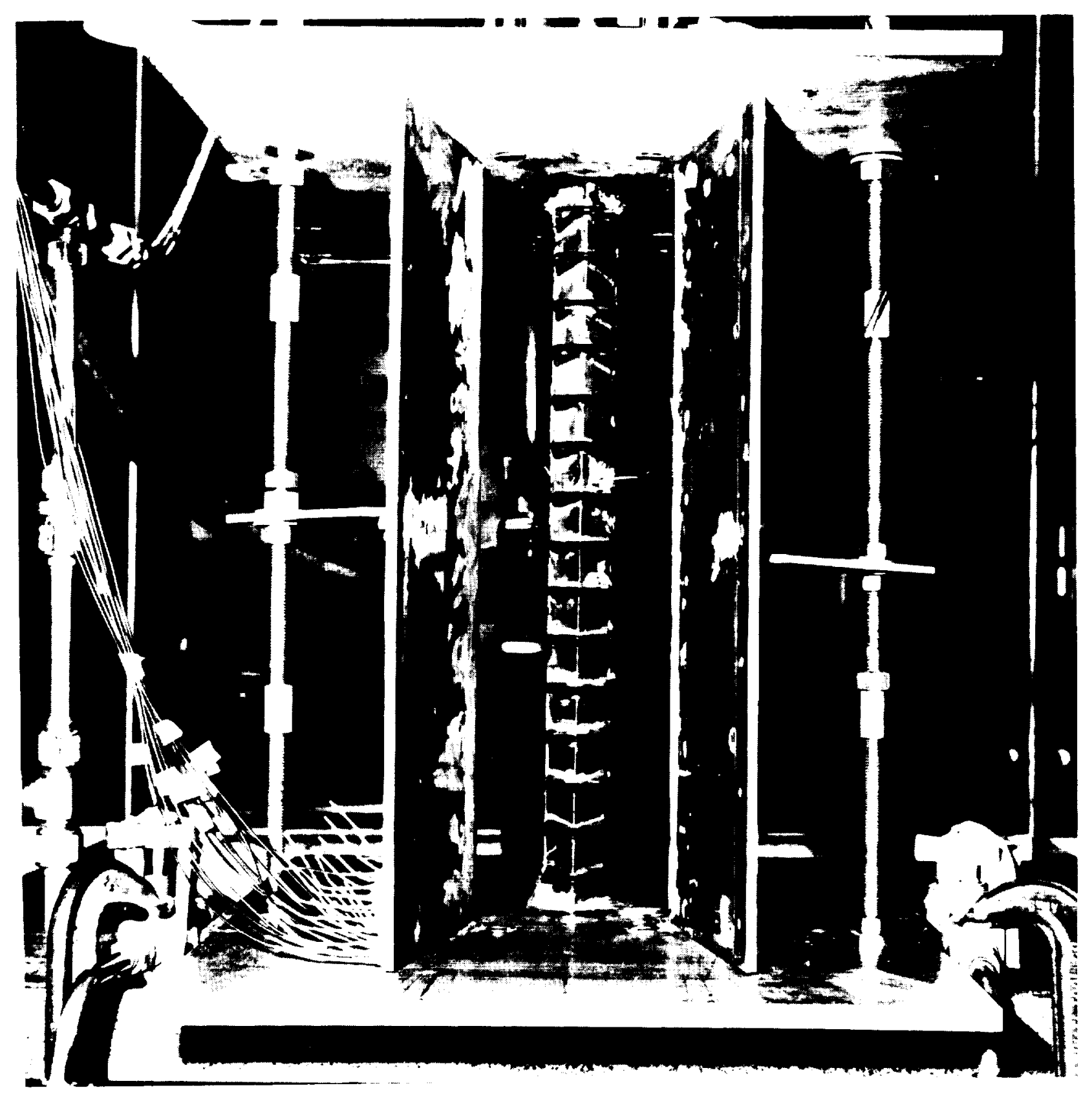

Figure 2. Test augmenter. 


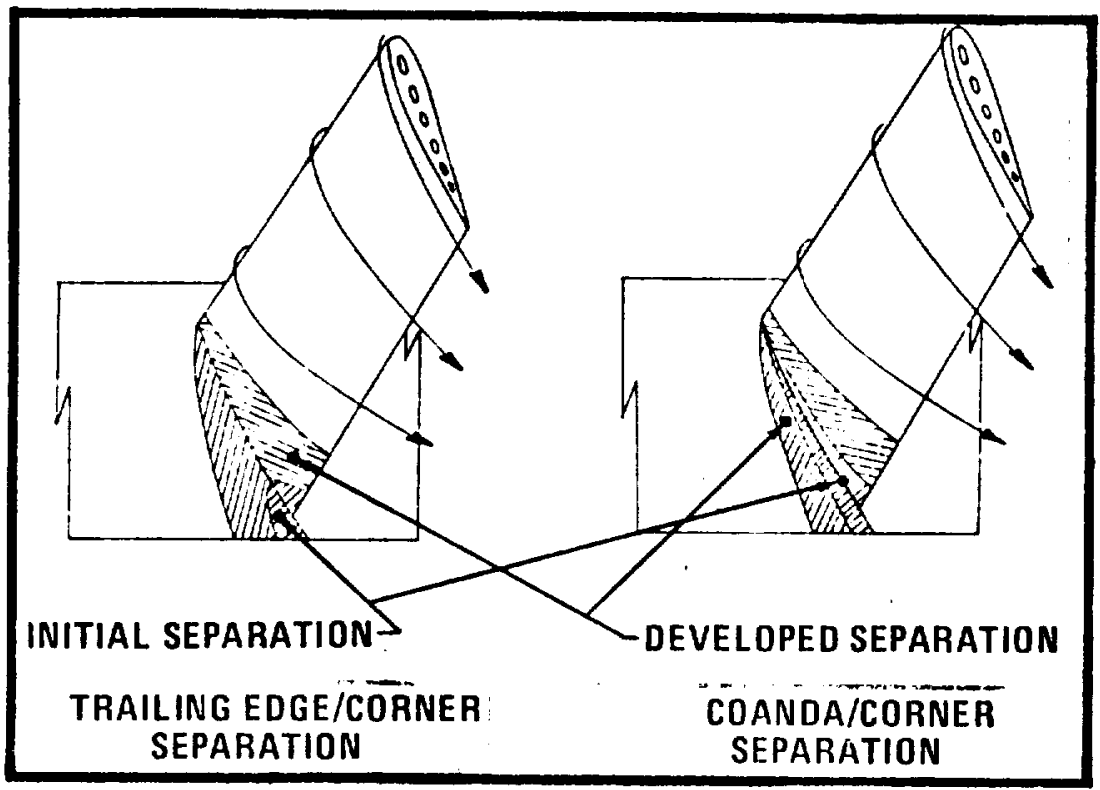

Figure 3.- Possible modes of Corner separation.

\section{(All Dimensions in Centimeters)}
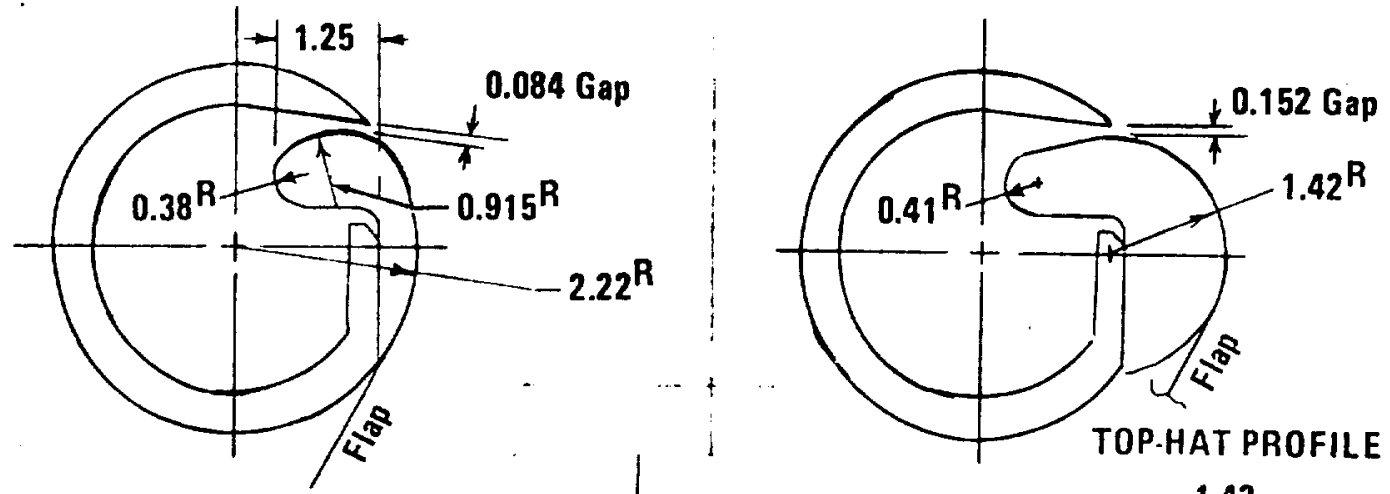

REFERENCE PROFILE

$R / t=2.22 / 0.084=26.5$

CAD 8973

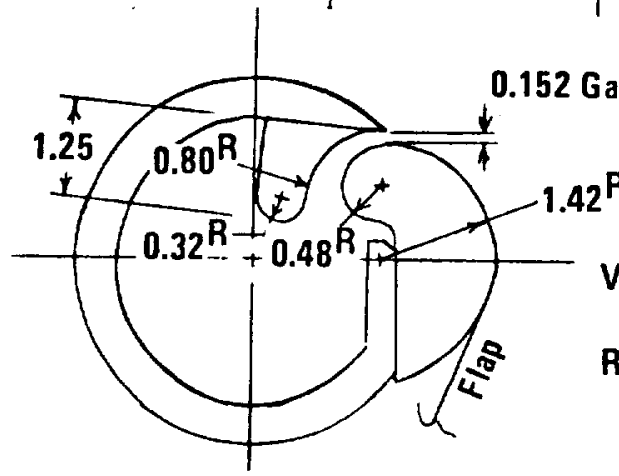

$$
R / t=\frac{1.42}{0.152}=9.3
$$

VORTEX PROFILE

$R / t=\frac{1.42}{0.152}=9.3$

Figure 4. Coanda nozzles tested in present study. 


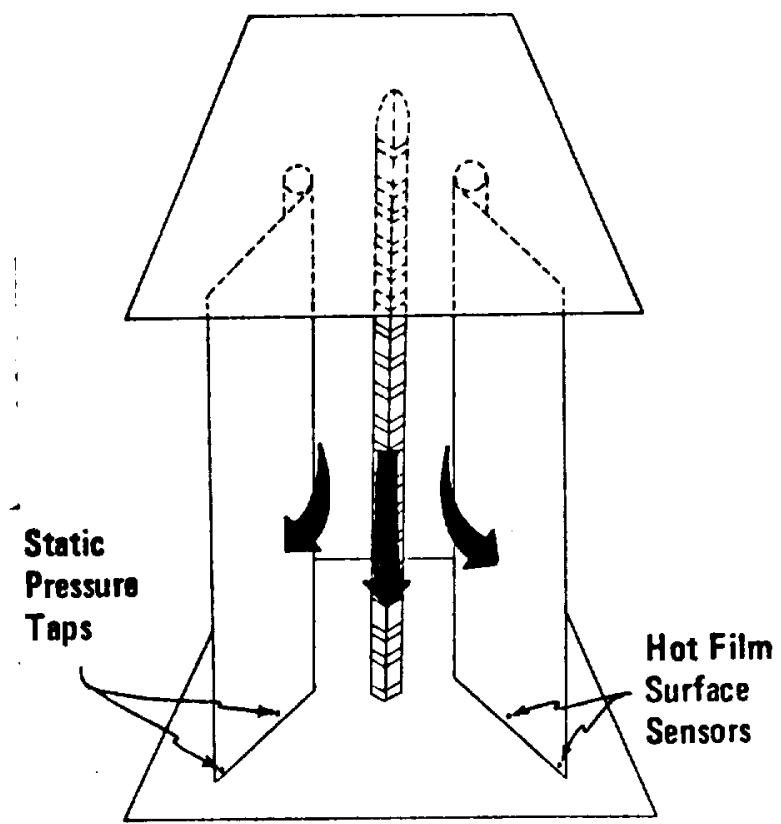

CAD $897 B$

Figure 5. Instrumentation for separation mode determination.

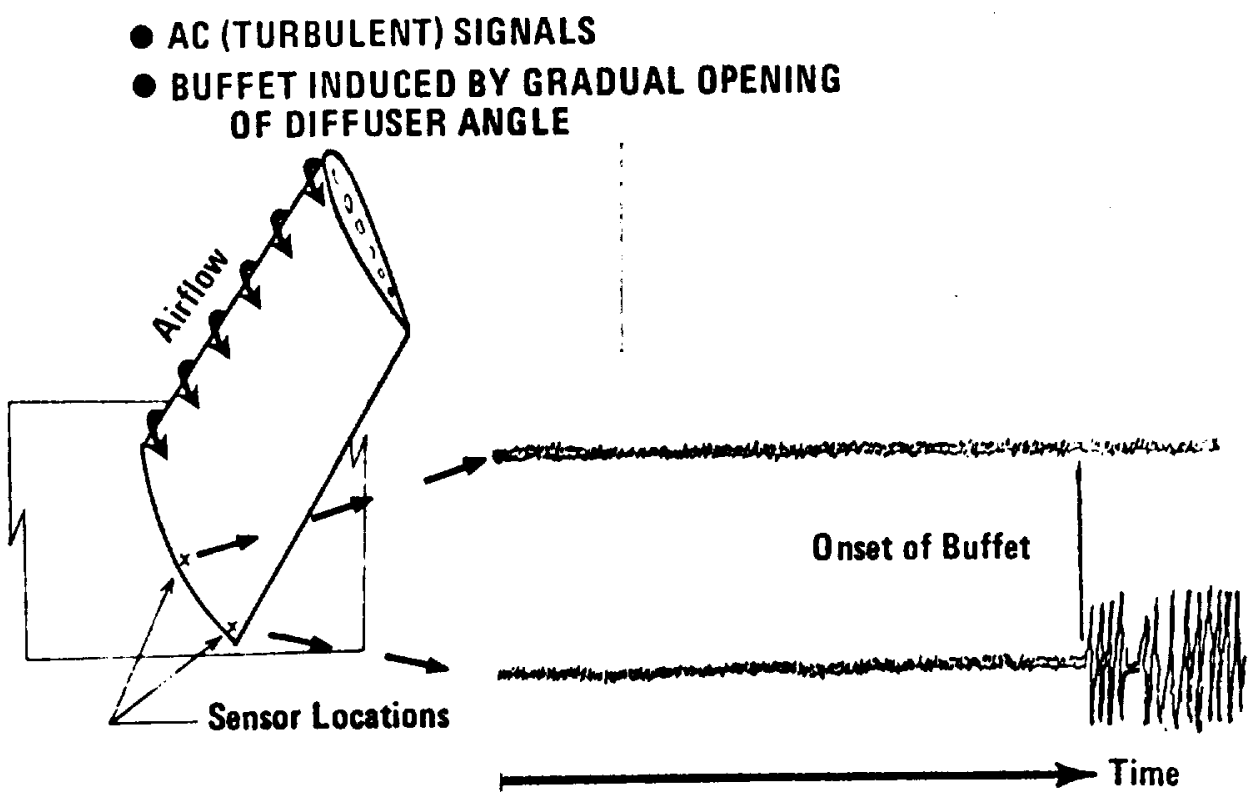

CAO 8977

Figure 6. Buffet response of hot film sensors: $P_{R}=2.0$. 


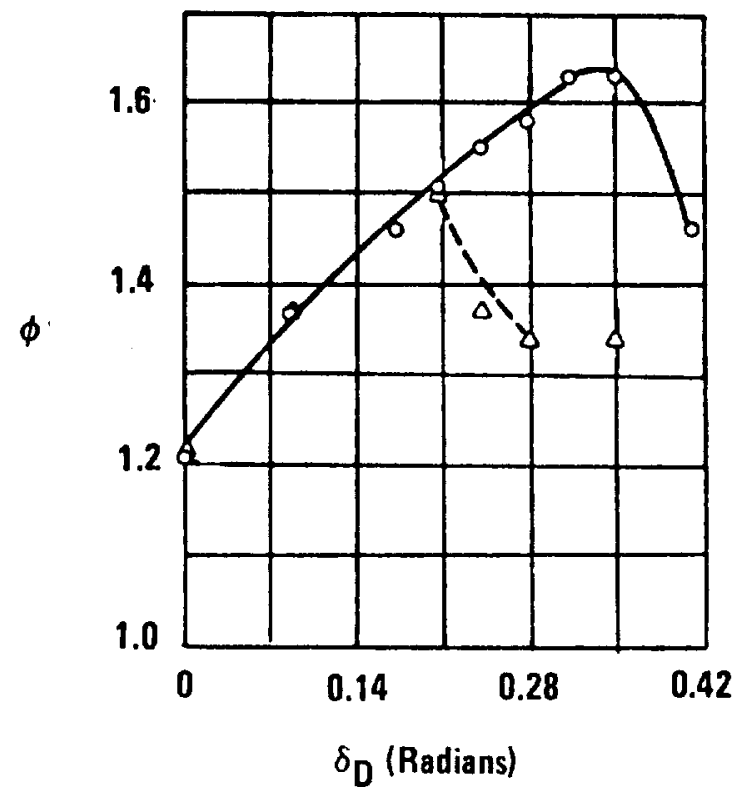

O Full BLC

$\triangle$ No BLC

Figure 7. Augmentation ratio vs diffuser angle: $A R=4.1$.

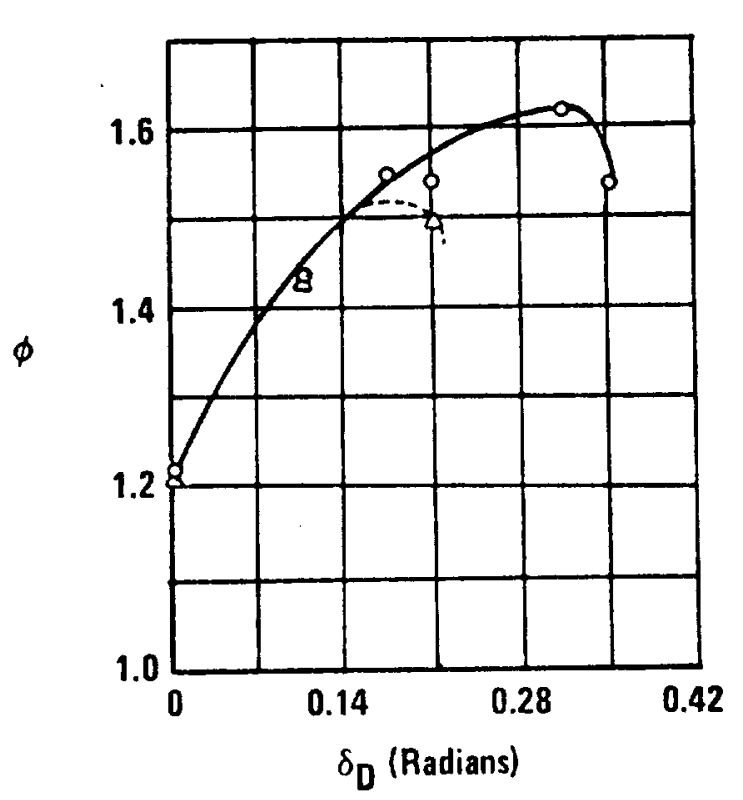

Full BLC

$\triangle$ No BLC

CAD 8978

Figure 8. Augmentation ratio vs diffuser angle: $A R=2.5$. 


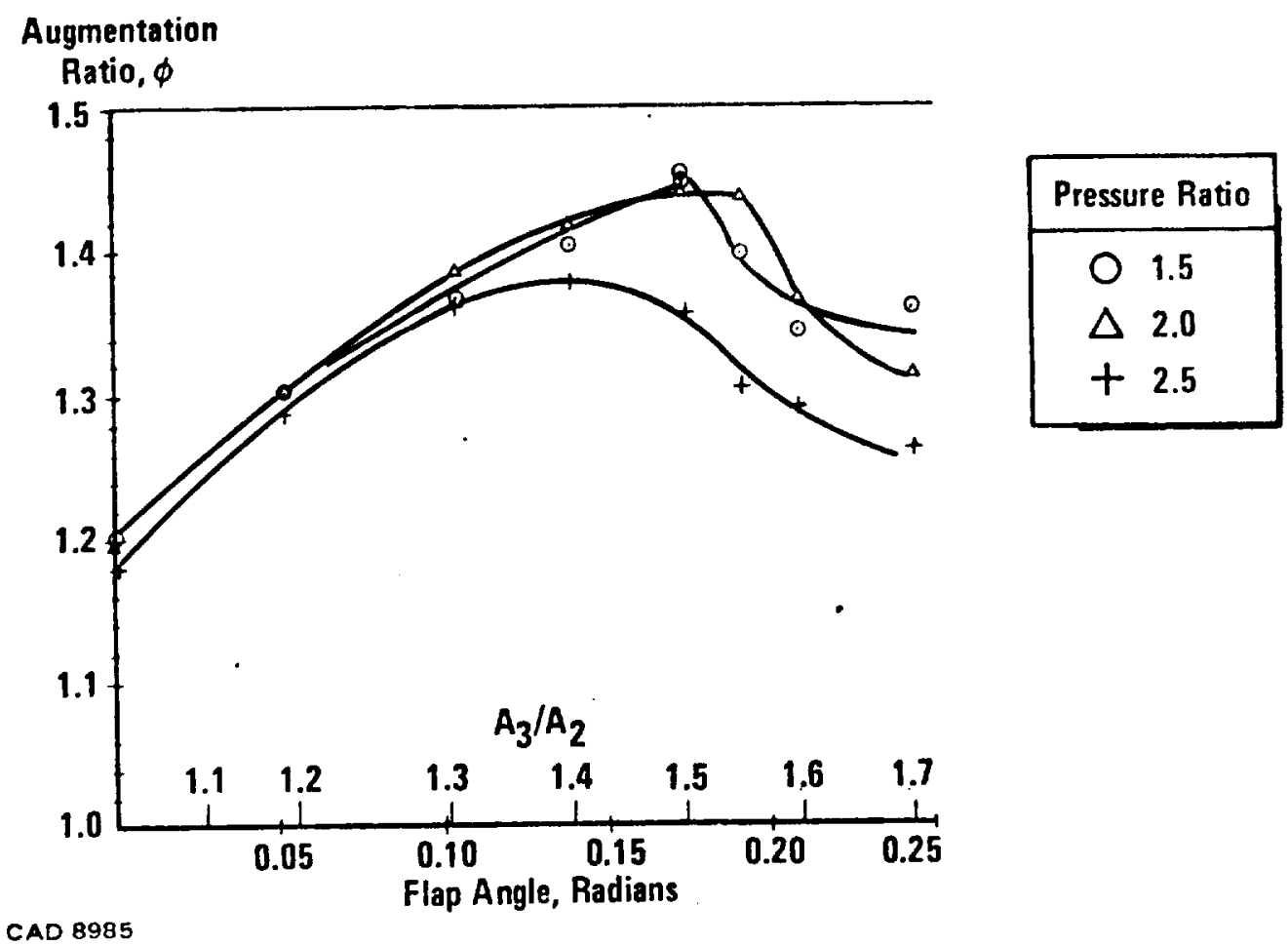

Figure 9. Augmentation ratio vs diffuser angle, top-hat profile.

\section{Augmentation}

Ratio, $\phi$

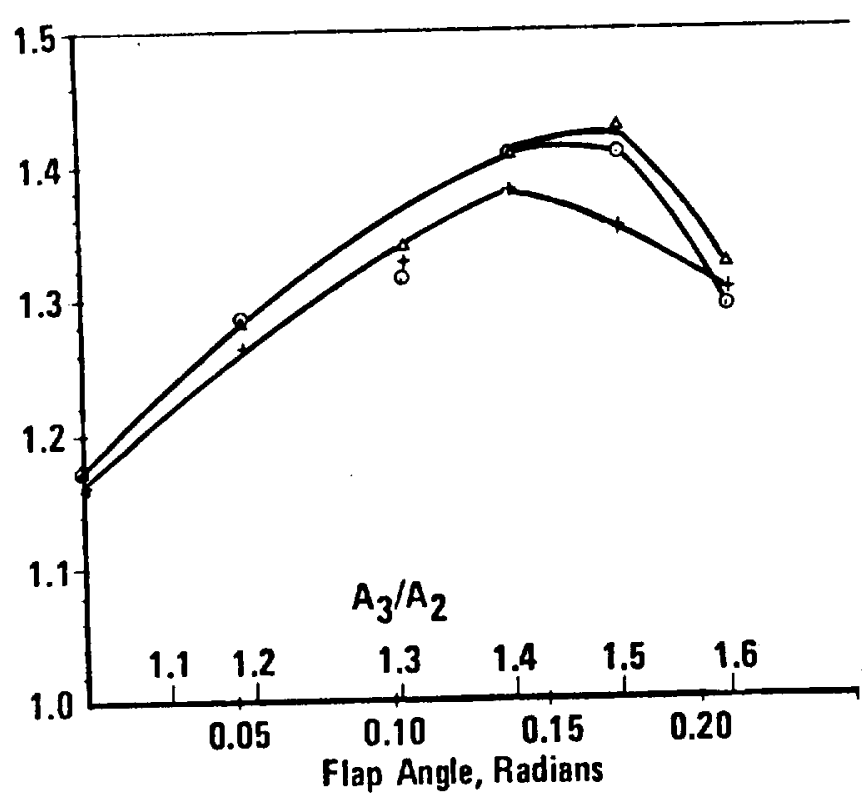

\begin{tabular}{|c|c|}
\hline Pressu & Ratio \\
\hline 0 & 1.5 \\
\hline$\Delta$ & 2.1 \\
\hline$x$ & 2.5 \\
\hline
\end{tabular}

CAD 8983

Figure lu. Augmentation ratio vs diffuser angle, vortex prufjle. 


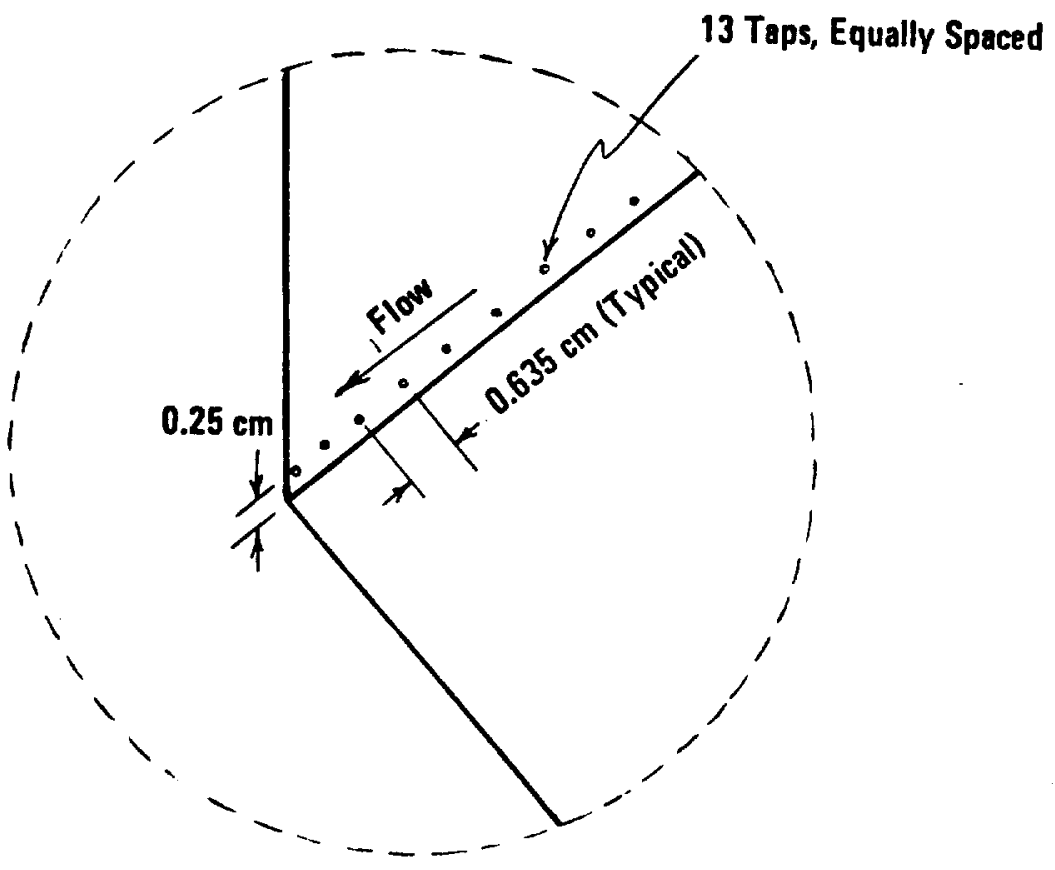

CAD 8979

Figure 11. Location of corner static pressure taps.

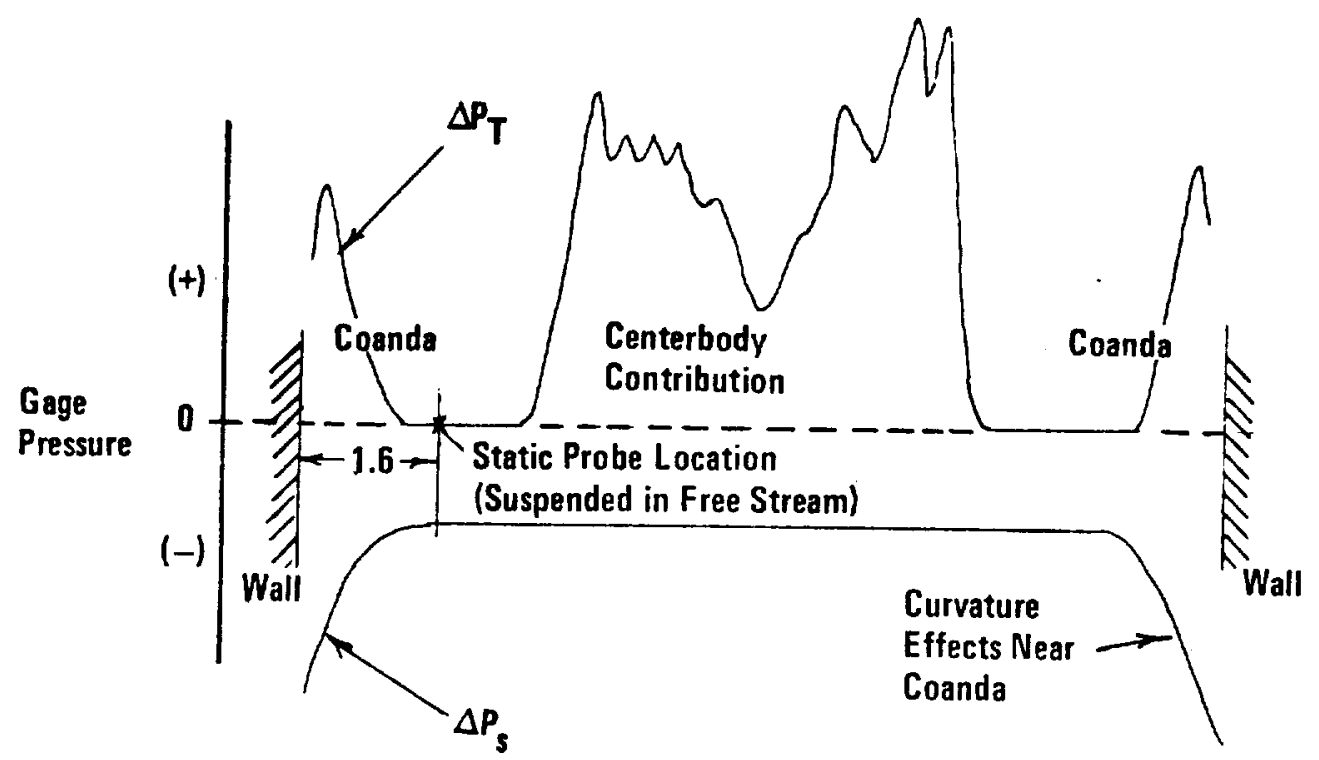

CAD 8980

Figure 12. Typical total, $\begin{aligned} \Delta \mathrm{P}_{\mathrm{T}} \text {, and static } \Delta \mathrm{P}_{\mathrm{S}} \text { distribution across the } \\ \text { augmenter throat. }\end{aligned}$ 


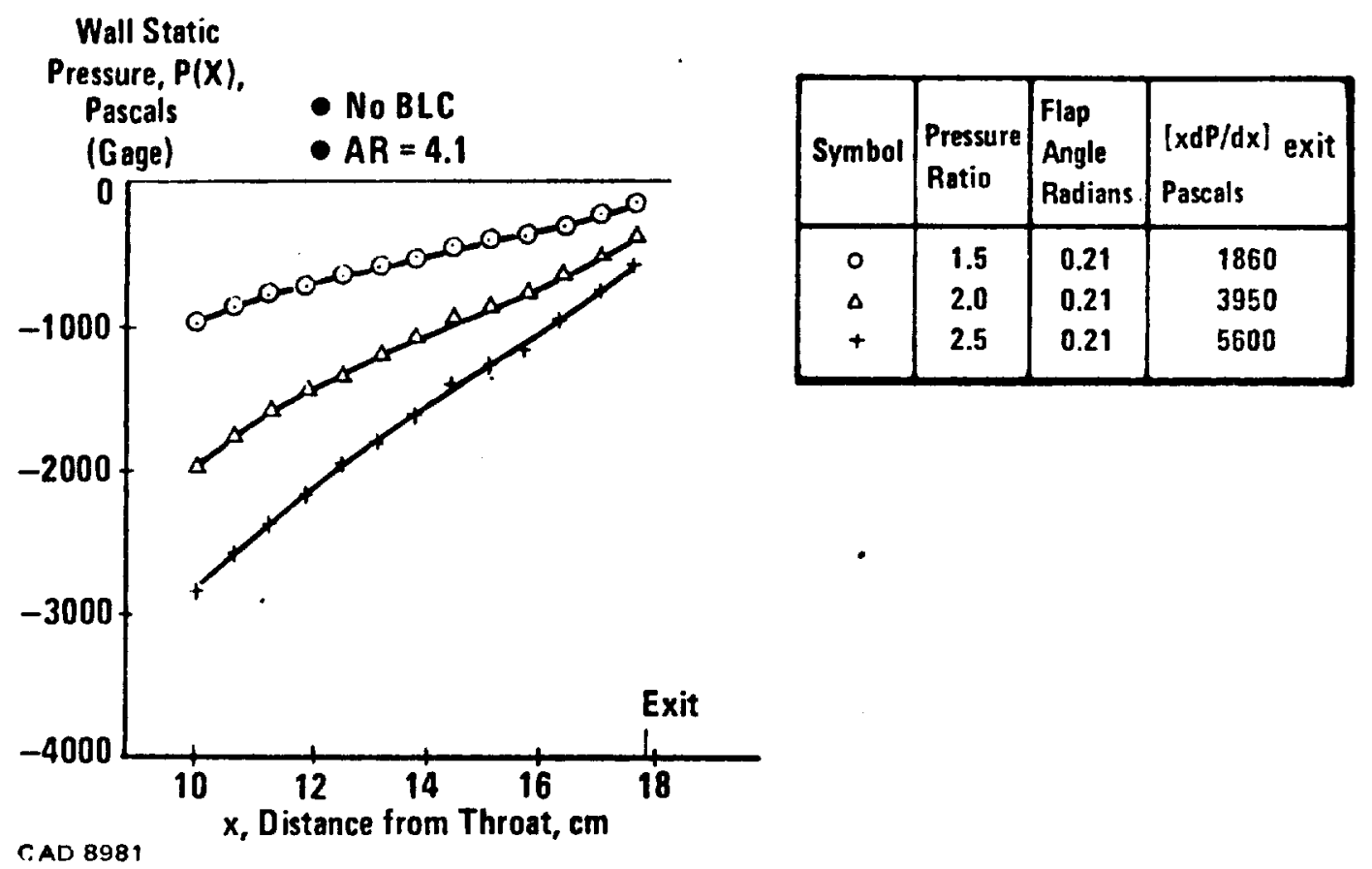

Figure 13. Corner static pressure readings for reference profile Coandas, $\mathrm{R} / \mathrm{t}=26.5$, no BLC.

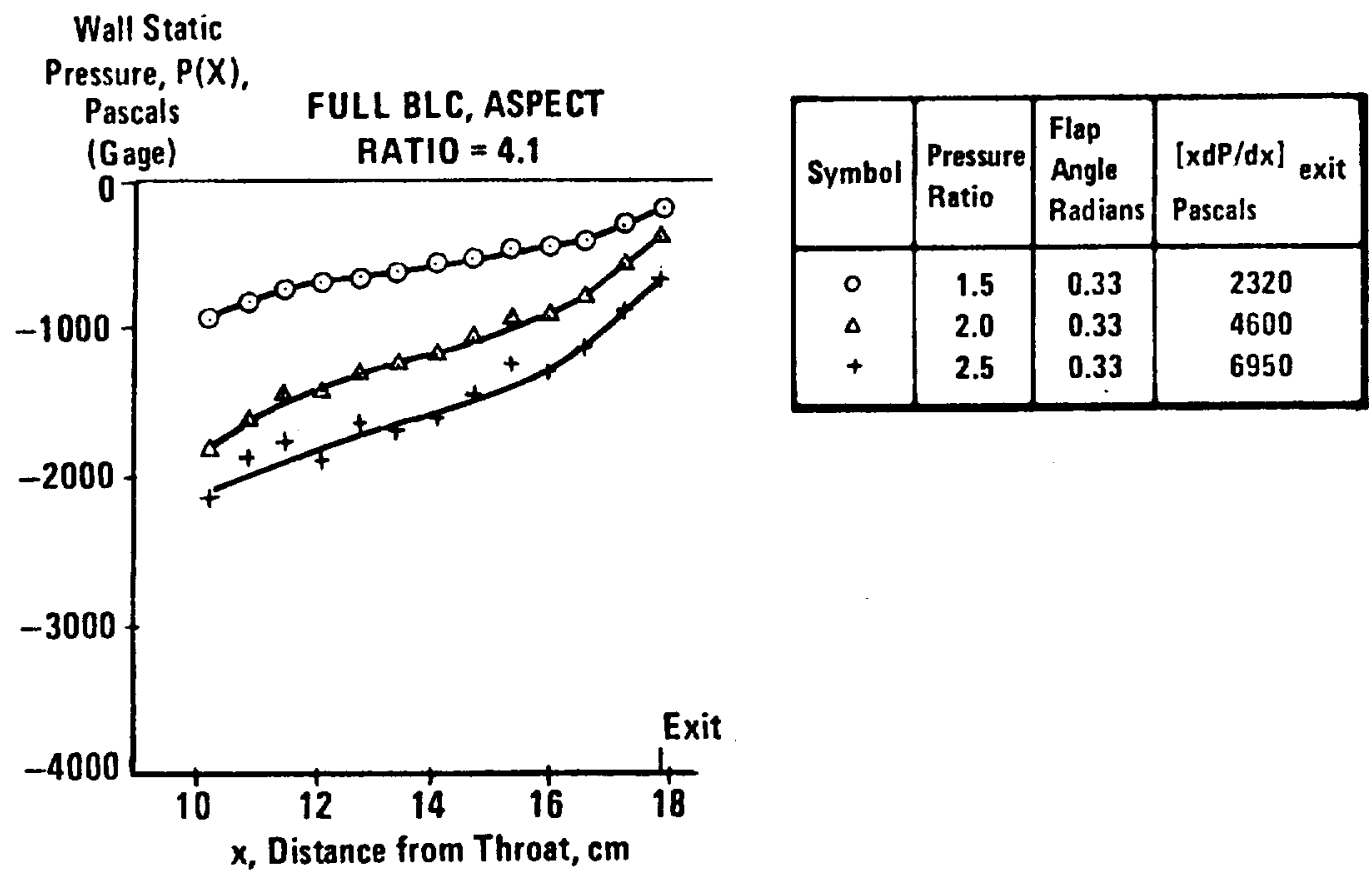

CAD 8982

liksure 14. Corner static pressure readings for reference profile Coandas, $R / t=26.5$, full BLC. 
Wall Static

Pressure, $P(X)$,

Pascals

(Gage)

\section{ASPECT RATIO $=4.1$}

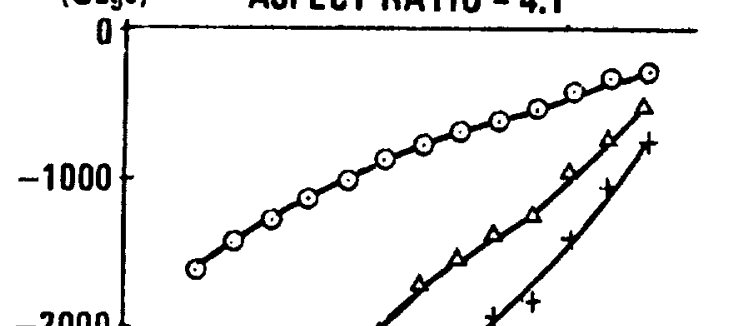

\begin{tabular}{|c|c|c|c|}
\hline Symbol & $\begin{array}{l}\text { Pressure } \\
\text { Ratio }\end{array}$ & $\begin{array}{l}\text { Flap } \\
\text { Angle } \\
\text { Radians }\end{array}$ & $\begin{array}{l}\text { [xdP/dx] exit } \\
\text { Pascals }\end{array}$ \\
\hline 0 & 1.5 & 0.175 & 2440 \\
$\Delta$ & 2.0 & 0.175 & 6500 \\
+ & 2.5 & 0.140 & 9750 \\
\hline
\end{tabular}

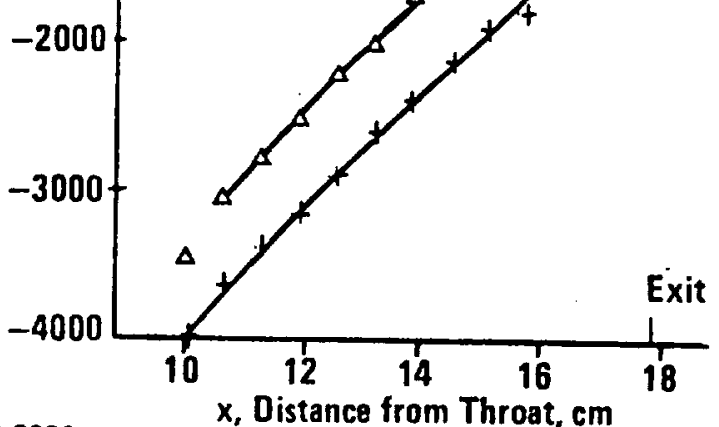

CAD 8986

$x$, Distance from Throat, $\mathrm{cm}$

Figure 15. Corner static pressure readings for top-hat profile, $\mathrm{R} / \mathrm{t}=9.3$, full BLC.

Wall Static

Pressure, $P(X)$,

Pascals

(Gage)

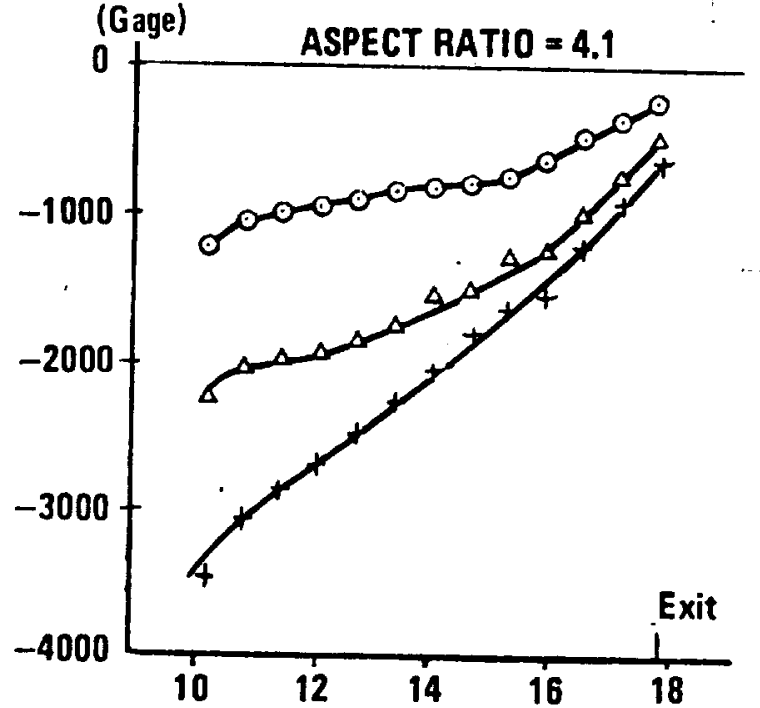

\begin{tabular}{|c|c|c|c|}
\hline Symbol & $\begin{array}{c}\text { Pressure } \\
\text { Ratio }\end{array}$ & $\begin{array}{c}\text { Flap } \\
\text { Angle } \\
\text { Radians }\end{array}$ & $\begin{array}{c}{[x \mathrm{dP} / \mathrm{dx}]_{\text {exit }}} \\
\text { Pascals }\end{array}$ \\
\hline$\circ$ & 1.5 & 0.175 & 3480 \\
$\frac{\Delta}{\mp}$ & 2.1 & 0.175 & 7000 \\
2.5 & 0.140 & 8850 \\
\hline
\end{tabular}

CAO 8984

$x$, Distance from Throat, $\mathrm{cm}$

Figure 16. Corner static pressure readings for vortex profile, $R / t=9.3$, full BLC. 


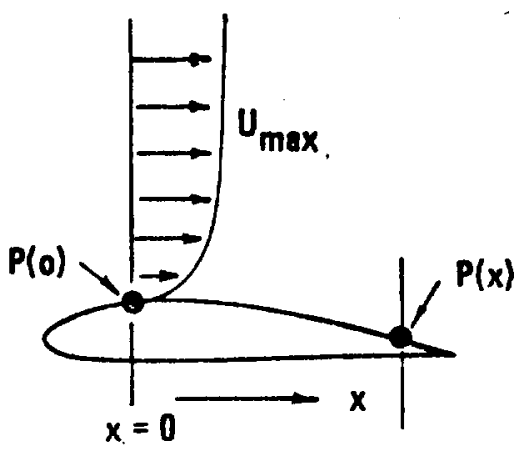

$N_{S T}=\frac{C_{P}\left(X d C_{P} / d x\right)^{1 / 2}}{\left(R_{N} \times 10^{-6}\right)^{1 / 10}}$

$C_{P}=\frac{P(x)-P(0)}{q(0)}$.

$q(0)=1 / 2 \rho U_{\max }^{2}$

$R_{N}$ from $U_{\max }$ and $x$ CAD 8987
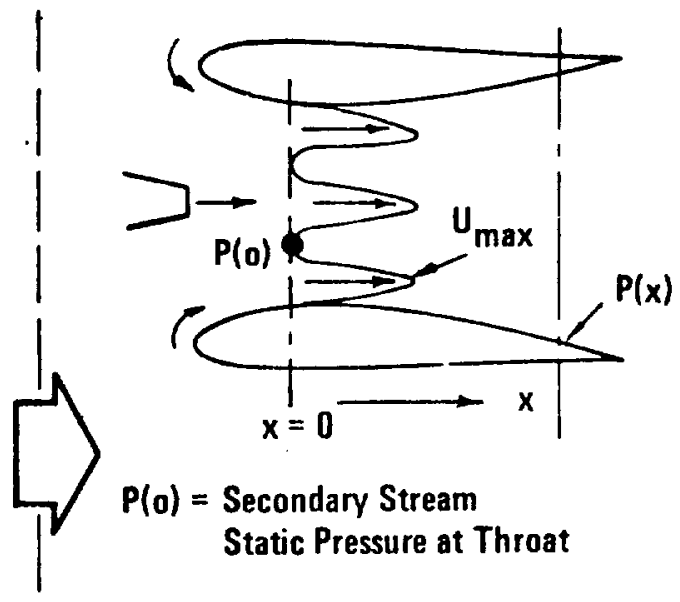

$P(0)=$ Secondary Stream Static Pressure at Throat

$q(0)=P_{T}-P_{\infty}$, Wall Jet or BLC Nozzle (Greatest)

$U_{\max }$ from $q(0)$

$R_{N}$ from $U_{\max }$ and $x$

Figure 17. Stratford and modified Stratford.

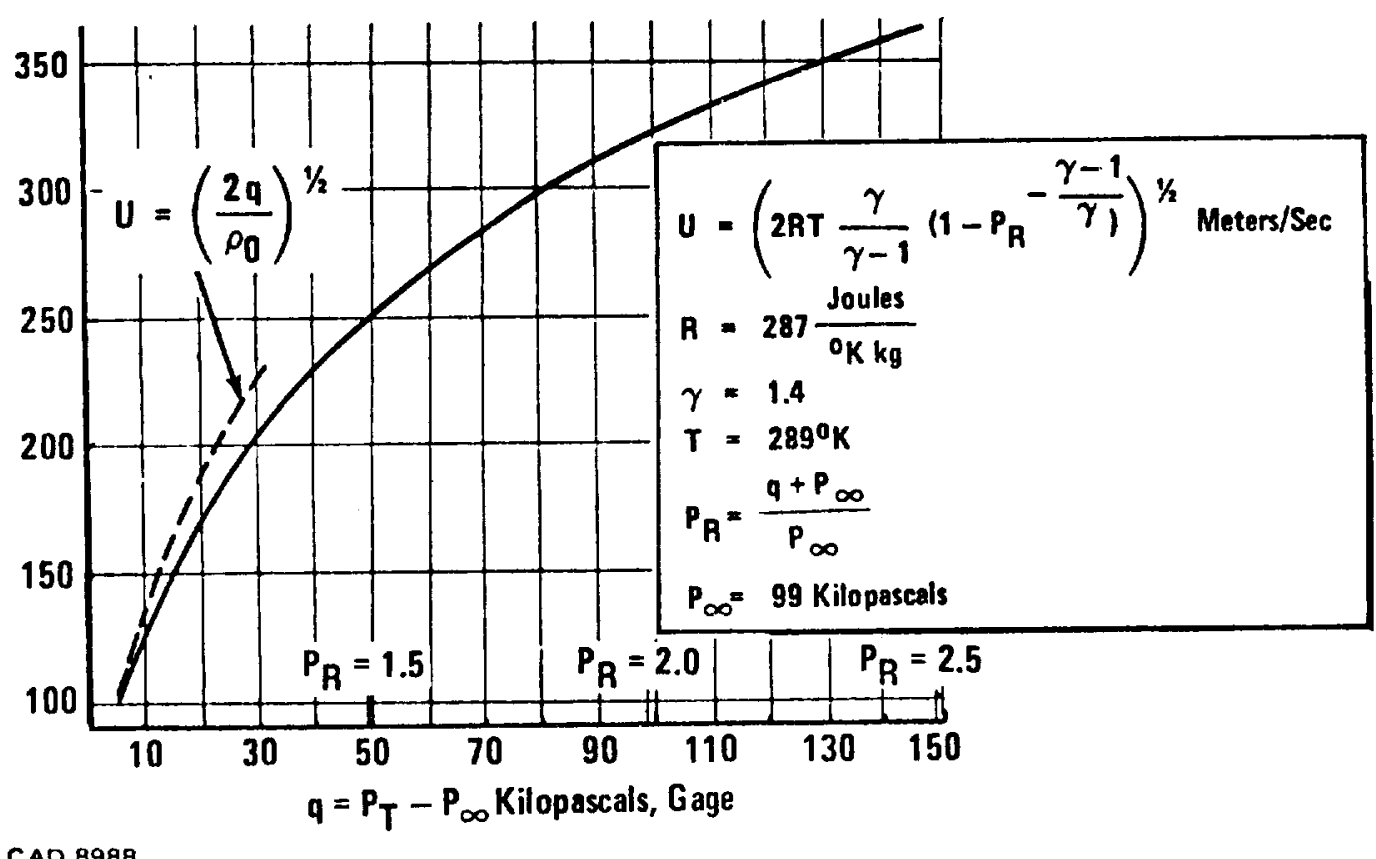

Figure 18. Velocity vs $q$. 


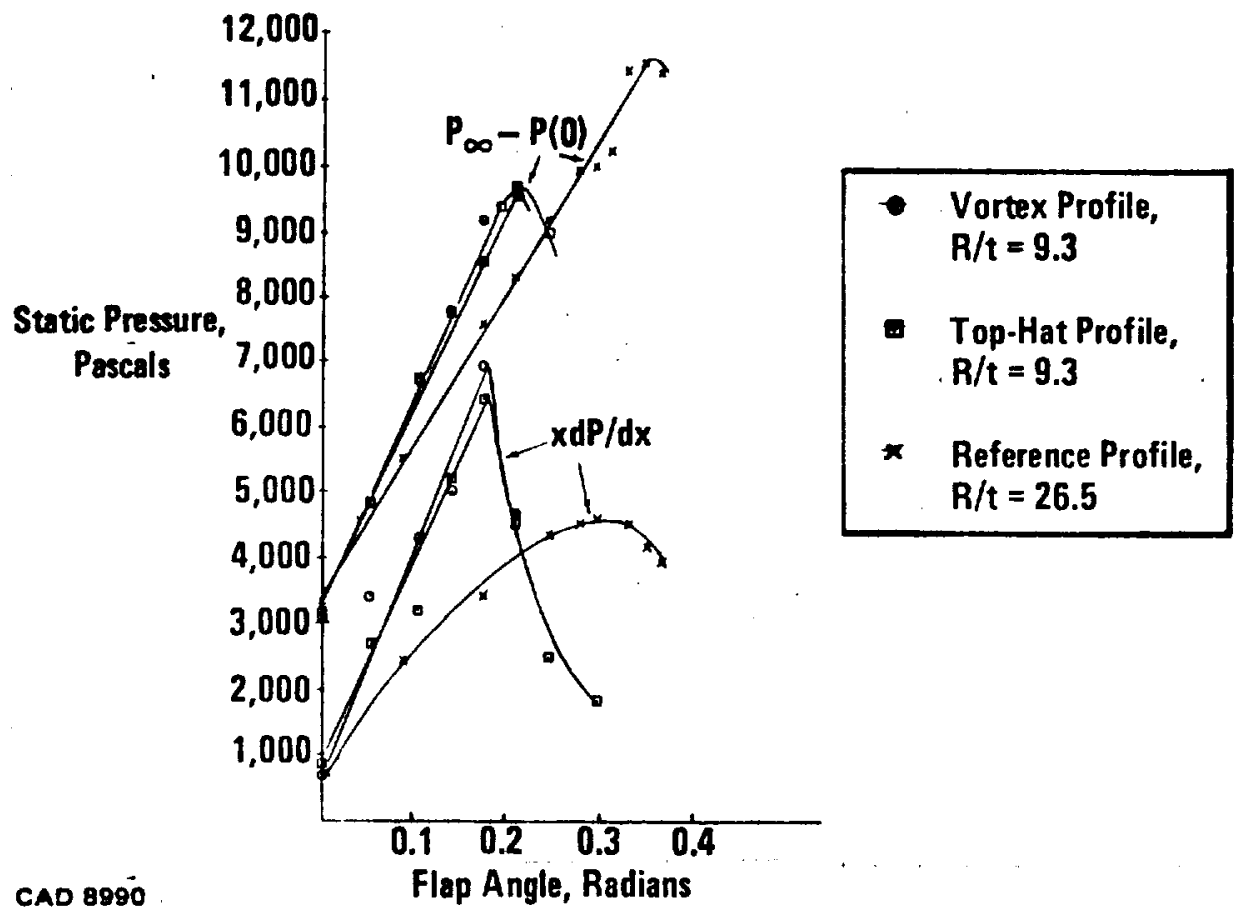

Figure 19. Variation of static pressure components with flap angle.

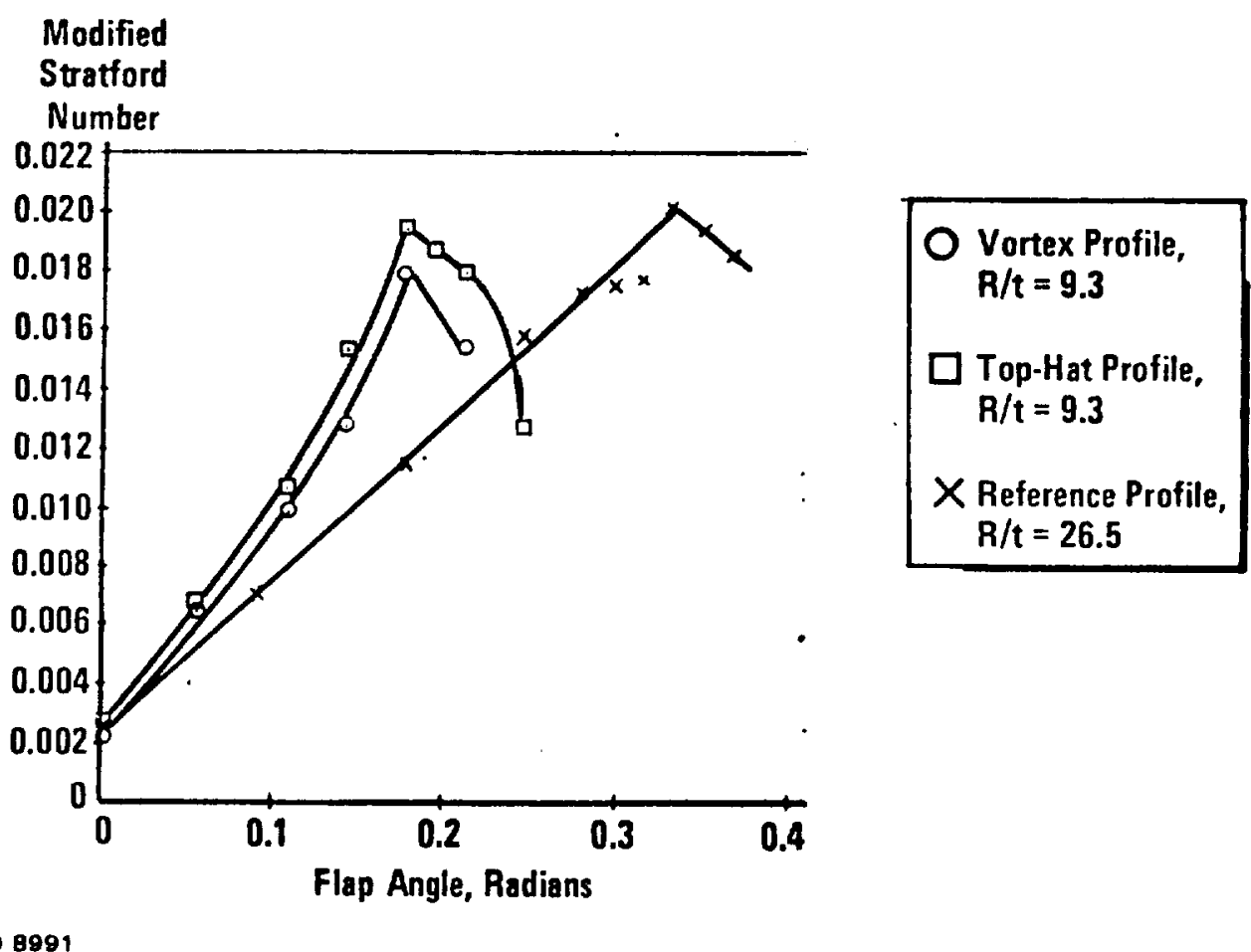

Figure 20. Variation of Stratford number with flap angle. 


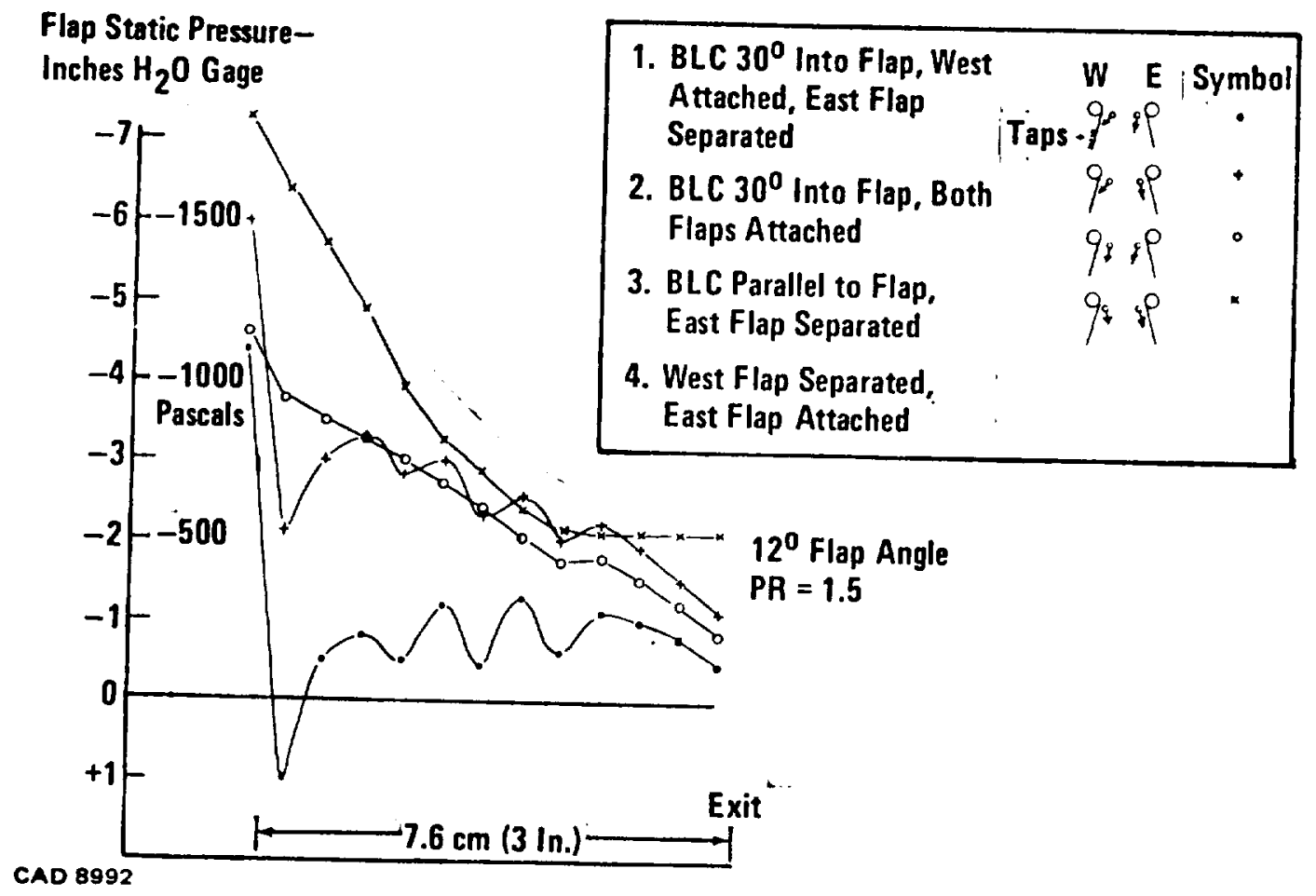

Figure 21. Effects of BLC nozzle orientation on flap statics. 